\title{
Sextant
}

Revue de recherche interdisciplinaire sur le genre et la sexualité

\section{"Pas d'enfant si je veux ». Stérilisée et sans enfant, un refus par corps de la maternité}

Emma Tillich

\section{OpenEdition}

\section{Journals}

Édition électronique

URL : https://journals.openedition.org/sextant/353

DOI : $10.4000 /$ sextant.353

ISSN : 2795-8736

\section{Éditeur}

Éditions de l'Université de Bruxelles

\section{Édition imprimée}

Date de publication : 1 décembre 2019

Pagination : 73-89

ISBN : 978-2-8004-1705-9

ISSN : $1370-267 X$

\section{Référence électronique}

Emma Tillich, « «Pas d'enfant si je veux ». Stérilisée et sans enfant, un refus par corps de la maternité », Sextant [En ligne], 36 | 2019, mis en ligne le 01 novembre 2021, consulté le 08 décembre 2021. URL : http://journals.openedition.org/sextant/353 ; DOI : https://doi.org/10.4000/sextant.353

\section{c) ()이 (2)}

La revue Sextant est mise à disposition selon les termes de la Licence Creative Commons Attribution Pas d'Utilisation Commerciale - Partage dans les Mêmes Conditions 4.0 International. 


\title{
«Pas d'enfant si je veux » Stérilisée et sans enfant, un refus par corps de la maternité
}

\author{
Emma Tillich
}

\section{Résumé :}

Cet article se penche sur la pratique de la stérilisation chez des femmes sans enfant par choix. Il décrit les ressources que ces femmes mobilisent pour obtenir une stérilisation, ainsi que les justifications qu'elles apportent à ce choix de contraception. La stérilisation fait l'objet d'un investissement symbolique très fort de la part des enquêtées : moyen d'auto-contrôle de sa faculté reproductive, il pourrait tout autant revêtir des caractéristiques "distinctives ». Pratique fortement féminisée, nous nous demanderons dans un dernier temps si elle ne constitue pas une normalisation des corps féminins.

\begin{abstract}
:
This article presents some ethnographic results about childfree women's voluntary sterilisation. It describes the resources that women mobilise to access sterilisation, as well as the justifications they produce to explain their contraceptive choice. Sterilisation is highly symbolic for these women: it is self-control of one's reproductive capacity but also a way to " distinguish » oneself. This practice is highly feminine, so we will finally discuss the possibility that it may be a form of normalisation of women's bodies.
\end{abstract}


« Comme je dis souvent, pour mes trente ans je me fais stériliser. Voilà. Bah ça a un sens pour moi quoi ! Y en a pour leur trente ans elles font un enfant, bah moi non. Moi je me fais stériliser (rires). C'est un peu comme un cadeau, quoi, un cadeau de trente ans ! » déclare une enquêtée sans enfant. La formulation contient sa dose d'ironie : puisqu'il faut faire le choix d'avoir des enfants à la trentaine, l'enquêtée choisit de ne jamais en faire, qui plus est à un âge symbolique, celui de la première maternité.

En France, les femmes sont peu à faire ce choix : seulement 4,2\% des femmes de 15 ans à 49 ans, françaises, vivant en France métropolitaine sont stérilisées (FECOND, INSERM-INED, 2013). Se faire stériliser volontairement sera compris ici comme une méthode de contraception définitive, mettant fin à la fertilité, et émanant d'un individu responsable légalement. L'acte se distingue donc de la contraception réversible (pilule, DIU, préservatif interne ou externe) et de la castration, en ce que le seul but est fonctionnel, et non pas punitif ou mutilatoire ${ }^{1}$.

Tout comme les femmes stérilisées, le choix de vie sans enfant est minoritaire en France, et moins déclaré par les femmes que par les hommes ${ }^{2}$. On estime l'infécondité volontaire en France à $5 \%$, chiffe stable depuis deux décennies ${ }^{3}$.

Aujourd'hui en France, la stérilisation est un choix que peut théoriquement faire tout individu, selon la loi du 4 juillet 2001 sur l'avortement et la contraception. À certaines conditions... Seul·e·s habilité $\cdot e \cdot s$ à la pratiquer, de nombreux'ses gynécologues s'opposent à la banalisation d'un acte pourtant libéralisé par le droit : «Que la stérilisation soit féminine ou masculine, son énorme charge psychosociale demeure et l'on aurait bien tort de penser que la récente libéralisation de la législation française pourrait être une incitation à moins de rigueur et de précautions dans les indications $"{ }^{4}$. Quatre mois de réflexion imposés par la loi de 2001 contribuent à la gravité perçue de l'acte, en imposant une temporalité longue pour la décision.

Qualifiée d'acte grave, la stérilisation reste un choix radical, qui donne une réalité corporelle au choix de non-maternité : pour une femme sans enfant, il s'agit d'un refus radical de donner naissance. Particulièrement négatifs à son égard, certain $\cdot e \cdot s$ médecins ou expert·e $\cdot s$ interrogé $\cdot e \cdot s$ dans les reportages en ligne sur le sujet ${ }^{5}$ évoquent $^{-}$ une mutilation, une " atteinte à la dignité de la personne ", et ce surtout quand la femme n'a pas eu d'enfants.

Le sujet de la stérilisation volontaire sans enfant a récemment connu une petite émergence médiatique. Dans la presse web ou papier, à la radio, des femmes sans enfant revendiquent leur " droit » à être stérilisées, tout comme on parle d'un droit à l'avortement ou à la contraception. Elles évoquent un « parcours du combattant

${ }^{1}$ A. Giami et H. Leridon, Les enjeux de la stérilisation, Paris, INED Éditions, 2000, p. 32.

${ }^{2}$ M. Mazuy, Ch. Debest et alii, « Rester sans enfant, un choix de vie à contre-courant », Population et Sociétés, ${ }^{\circ}$ 508, 2014.

3 Ibid.

${ }^{4}$ P. Madelenat, « Introduction », in B. Blanc et P. Madelenat, La stérilisation à visée contraceptive, Paris, Elsevier Masson, 2004, p. 5.

${ }^{5}$ Voir le web-documentaire « J'ai décidé d'être stérile » (2015) (https://www.lesinrocks. com/inrocks.tv/jai-decide-detre-sterile-le-webdocumentaire-sur-la-sterilisation-volontaire-desfemmes-qui-ne-veulent-pas-etre-meres/) 
pour accéder à la stérilisation ». L'intuition selon laquelle ces femmes feraient l'objet d'un « cadrage déviant ${ }^{6}$ de la part de la profession médicale est confirmée par les résultats de notre enquête de terrain pour notre mémoire de Master 1. Les médecins sont régis par un ensemble de normes explicites mais aussi implicites encadrant la pratique de la stérilisation et tenant pour beaucoup aux normes procréatives : une femme « stérilisable » répond à un certain nombre de critères, dont les principaux sont l'âge et le nombre d'enfants. En dessous de 30 ans et de deux enfants, la majorité des praticiens refuse de pratiquer l'acte.

Nous avons donc affaire, d'un côté, à un acte peu pratiqué, tardivement régi par le droit, et dont la réalisation sur une femme sans enfant semble constituer une déviance. De l'autre côté, comme l'illustre la citation mise en exergue de cet article, l'acte revêt cependant, pour une population bien spécifique, une haute valeur symbolique. Comment les enquêtées revendiquent-elles dans ce cas un choix considéré comme « déviant » par les médecins - mais pas que ? Cela constitue-t-il un défi des normes procréatives et contraceptives ?

Nous répondrons à cette question de trois manières : premièrement en décrivant les ressources qui permettent aux femmes de revendiquer leur stérilisation. Deuxièmement, nous nous attacherons aux régimes de justification qui entourent la stérilisation. Enfin, nous nous centrerons sur les valeurs qui sont associées à la stérilisation : pourquoi prend-elle une telle importance pour les childfree?

\section{Encadré 1 : matériaux et méthodes}

Nous avons mené une enquête essentiellement ethnographique, auprès de gynécologues pratiquant ou non la stérilisation (cinq gynécologues exerçant en clinique ou en hôpital public ont été interrogés), ainsi qu'auprès de femmes stérilisées ou aspirant à l'être (onze femmes sans enfant ont été interrogées, ainsi que deux femmes ayant eu des enfants). Nous avons pu observer deux consultations pour stérilisation en suivant une enquêtée dans son parcours.

Notre ethnographie s'est poursuivie en ligne, par l'observation systématique des publications de divers groupes Facebook childfree (personnes se revendiquant sans enfant par choix), dont un nombre conséquent traite du sujet de la stérilisation volontaire.

Nous avons doublé cette enquête ethnographique de la consultation de la littérature médicale au sujet de la stérilisation.

\section{Stérilisée sans enfant : les ressources d'un choix de vie}

Qui sont ces femmes sans enfant, et quels moyens mettent-elles en œuvre pour accéder à la stérilisation?

\section{Femmes sans enfants : déviantes ou dominantes?}

Les enquêtées appartiennent aux catégories moyennes à moyennes supérieures, ce qui concorde avec l'appartenance sociale de la population sans enfant volontaire

${ }^{6}$ R. Dingwall et T. Murray, « Categorization in accident department: "Good” patients, "Bad" patients and "Children" », Sociology of Health and Illness, 5 (2), 1983, p. 129. 
selon les travaux sur le sujet ${ }^{7}$. Souvent employées dans le secteur privé ou tertiaire, trois étaient cadres en entreprise, une autre professeure des écoles, deux secrétaires médicales, deux à leur compte dans le secteur tertiaire. Une autre suivait des études d'aide-soignante, une était sans-emploi diplômée d'un master d'Histoire, une autre encore était chauffeure poids-lourd. Elles avaient entre 20 et 51 ans au moment de l'enquête. Ces « déviantes »-aux yeux des médecins mais aussi au regard des normes procréatives - appartiennent donc à des catégories disposant des « armes sociales permettant de refuser la maternité $\gg{ }^{8}$, ainsi que de remettre en cause la hiérarchie implicite des moyens de contraception imposée par le corps médical. Ces armes sociales sont le capital culturel, qui permet, nous le verrons, de savoir justifier son choix et de savoir où chercher des informations alternatives. Un certain capital économique, aussi, permettant d'assumer les éventuels coûts de ce « parcours du combattant » pour trouver un gynécologue : déplacements, dépassement d'honoraires, etc.

Plus que cela, les femmes interrogées et plus largement les childfree sont une communauté qui présente des valeurs particulières.

\begin{tabular}{|l|}
\hline Encadré 2 : le mouvement childfree \\
Le mouvement childfree, ou « sans enfant par choix » est un mouvement regroupant des \\
personnes revendiquant un choix de vie sans enfant. Il s'agit d'un mouvement, sinon militant, \\
de revendication identitaire qui s'appuie sur la distinction entre childless (personnes n'ayant \\
pas d'enfants pour des raisons extérieures à leur volonté) et childfree (sans enfant par volonté \\
délibérée). Le mouvement est né en Amérique du Nord, avec l'Organisation Nationale des \\
Non-Parents (N.O.N), fondée en 1972 à Palo Alto, suivi par la création d'autres mouvements \\
comme No Kidding!International créé à Vancouver, au Canada par Jerry Steinberg. Elle \\
s'étend dans plusieurs pays anglophones et organise des événements et rencontres childfree. \\
En Europe, la mouvance childfree se développe majoritairement dans les années 2000, et \\
touche d'abord l'Angleterre, avec la création de Kidding Aside, association fondée en 2000 \\
afin de lutter pour les droits des personnes childfree. La Fête des Non-Parents, créée en 2009 \\
par l'écrivain antinataliste Théophile de Giraud et la musicologue Frédérique Longrée, a lieu \\
depuis en alternance à Paris et à Bruxelles.
\end{tabular}

Les justifications associées au choix de ne pas procréer sont généralement l'absence de désir, premièrement, assorti d'une série d'autres raisons, hédonistes ou présentées comme altruistes, comme le fait de ne pas vouloir « participer à la surpopulation » (motivations de type écologistes), ou plus rarement de ne pas vouloir transmettre de maladies génétiques.

F9 : Bah moi des enfants j'en veux pas parce que j'en veux pas. Point. J'ai pas le désir, quoi ?

$\mathrm{Q}$ : C'est avant tout une histoire de désir personnel donc?

F9 : Ben oui. Et puis aussi moi j'ai des raisons écologiques, quoi, je pense que c'est pas une bonne idée de faire des enfants avec tout ce qu'on fait à la terre... C'est égoïste, enfin si on veut parler d'égoïsme ! Les nanas qui font quatre, cinq, dix gosses...

${ }^{7}$ Ch. Debest, Le choix d'une vie sans enfant, Rennes, Presses Universitaires de Rennes (coll. «Le sens social »), 2014, p. 58 ; A. Gotman, Pas d'enfant. La volonté de ne pas engendrer, Paris, Éditions de la Maison des Sciences de l'Homme, 2017.

${ }^{8}$ Ch. Debest, Le choix d'une vie sans enfant, op. cit. 
$\mathrm{Q}:$ C'est pas responsable?

F9 : Ben non. Et puis non bah avec ma maladie moi je pense pas que c'est une idée franchement, je sais pas si j'ai vraiment envie de transmettre ça, enfin comme je disais.

F12 : La liberté c'est mon premier motif. Pouvoir voyager si je veux, pouvoir mettre des sous de côté pour réaliser mes projets. Parce que j'ai pas envie qu'un enfant vienne bousiller mon couple quoi. Enfin je sais qu'il y a des couples qui vivent très bien avec mais pour moi c'est un tue-l'amour. Que ça fait du bruit et que le bruit je le supporte de moins en moins. Que c'est contraignant, qu'il faut toujours être dispo. Quand on a un enfant il faut lui consacrer tout son temps, limite le faire passer avant soi-même.

Sont ainsi particulièrement valorisées l'indépendance, l'autonomie, la liberté (F12), la flexibilité du mode vie sans enfant, qui permet de réaliser voyages et activités, et plus important de se réaliser d'une manière présentée comme totalement personnelle - contrairement selon elles à la procréation. Tout permet de rapprocher les childfree de l'individu type de la « cité par projets », ce monde social du réseau et de la communication émergeant avec le « nouvel esprit du capitalisme », décrits par les sociologues L. Boltanski et E. Chiapello. Y sont valorisés l'enthousiasme, l'autonomie, l'employabilité, la connexion aux autres ${ }^{9}$.

\section{Les moyens de se faire entendre : accéder à la stérilisation}

L'accès à la stérilisation est très fortement régulé par la profession médicale avec pour critères principaux l'âge et de nombre d'enfants ${ }^{10}$. Ceux-ci sont prescrits par la littérature médicale mais surtout transmis de manière informelle par les pairs médecins lors des prises de décisions collectives. Face à ces barrières, les femmes adoptent des stratégies que nous allons décrire.

\section{Une utilisation alternative de l'information}

La première d'entre elles consiste en l'accès à une information alternative provenant majoritairement d'Internet, qu'elle soit issue de documentaires sur le sujet de la stérilisation ou de sites d'information sur la santé. Sont mentionnées également des listes de noms de gynécologues aux pratiques « féministes » ou ouvertes au sujet de la stérilisation ${ }^{11}$.

Le regroupement en une communauté partageant les mêmes valeurs et aspirations permet un meilleur accès à l'information. Certain $\cdot e \cdot s$ sans enfant par choix se retrouvent en ligne, sous le nom de childfree afin d'échanger expériences et conseils mais surtout de revendiquer leur choix de vie. Depuis quelques années, les childfree utilisent largement Facebook. Le plus grand groupe childfree francophone sur Facebook regroupe près de 1000 membres, certains groupes anglophones plusieurs milliers de membres. Le principal groupe childfree enquêté est un univers

${ }^{9}$ L. Boltanski et E. Chiapello, Le nouvel esprit du capitalisme, Paris, Gallimard, p. 625.

${ }^{10}$ D'après notre mémoire de Master 1 sur le sujet.

${ }^{11}$ Par exemple https://gynandco.wordpress.com/ et http://www.martinwinckler.com/spip. php?rubrique8. 
assez homogène de valeurs partagées, ce qui est surprenant au vu des différents mouvements combinés : « anti-natalisme », " écologie », " individualisme ». Il opère un syncrétisme de ces idéologies, garanti par une charte interdisant les débats entre différents mouvements. Témoin de la puissance socialisatrice de ces groupes, ce syncrétisme se retrouve dans les discours, souvent très similaires, des childfree interrogées. La plupart des membres présentent ces groupes Facebook comme des « refuges » virtuels, où des gens ayant pour point commun de ne pas vouloir d'enfant se réunissent pour partager leur "sentiment de décalage », ou parfois échanger des conseils pratiques quant à la stérilisation - déroulement d'une consultation pour stérilisation, listes de noms de praticiens, conseils variés, comme ceux de F11 sur la meilleure manière de justifier une stérilisation. Des parrainages informels existent, comme F12, qui, devenue amie avec une femme lui ayant demandé des conseils sur la stérilisation, l'a accompagnée dans son parcours. Les childfree utilisent également d'autres groupes sur la stérilisation, où la règle est le « non-jugement » de toutes les demandes de stérilisation.

Les exemples familiaux et amicaux constituent une dernière source d'information. La stérilisation d'une sœur ou d'une amie fait entrer l'acte jusque-là impensable dans l'ordre des possibles : trois des femmes childfree interrogées ont commencé à réfléchir à cet acte pour elles-mêmes après avoir vu un exemple dans leur entourage, comme F4 (29 ans, sans enfant), dont la mère a été stérilisée.

\section{Convaincre : une relecture de l'interaction médecin-patiente}

Face à une demande de stérilisation, les médecins agissent selon deux modes de 《 gouvernement des conduites corporelles $»^{12}$ - le terme désigne selon la sociologue D. Memmi la manière dont la société, et plus particulièrement les institutions, interviennent sur les corps et les façonnent selon un ensemble de « normes et de valeurs, de rapport d'autorité et de légitimité ${ }^{13}$. Le premier pourrait être qualifié d'autoritaire : il est représenté par un refus pur et simple de stérilisation. Le deuxième correspond à un mode de « gouvernement par la parole ${ }^{14}$ de la conduite des femmes : ce mode de gouvernement passe par le discours, le dialogue, plus largement l'interaction entre le médecin et la patiente. Le but, du moins affiché, n'est ici pas tant de prendre la bonne décision pour la patiente que de lui faire comprendre la décision du médecin : cela passe souvent par un dialogue rentrant avec précision dans les détails biographiques de sa vie. Ici, il s'agit d'un entretien aux tonalités psychologiques, visant à évaluer le risque de regret de la patiente après l'intervention.

Face à ces médecins tentant de gouverner leur conduite, les femmes se montrent fortement résistantes. Elles relisent l'interaction en termes agonistiques, parlent de « convaincre » le praticien, de se faire entendre, autant de registres évoquant une lutte pour la reconnaissance ${ }^{15}$. À aucun moment dans le discours des enquêtées n'est

${ }_{12}$ D. Memmi, Faire vivre et laisser mourir. Le gouvernement contemporain de la naissance et de la mort, Paris, La Découverte, 2003, p. 136. 2004.

13 D. Memmi et D. Fassin (dir.), Le gouvernement des corps, Paris, Éditions de l'EHESS,

14 D. Memmi, Faire vivre et laisser mourir, op. cit., p. 136.

15 A. Honneth, La lutte pour la reconnaissance, Paris, Folio, 2013. 
convoquée cette figure connue du gynécologue confident de la vie intime des femmes, guidant et orientant les conduites reproductives ${ }^{16}$. Nous pouvons le relier à la forte stigmatisation dont leur choix fait l'objet de la part de la profession médicale, mais aussi à une décision déjà affirmée et conçue comme purement personnelle. Elles défendent leur décision grâce à un corpus de justifications travaillées à l'avance. Une stérilisation acceptée par le gynécologue est parfois lue comme une victoire, due à des qualités de persuasion personnelles. F11, stérilisée à 19 ans après trois avortements affirme : « j’étais sûre de mon choix, j'ai bien expliqué et le médecin a accepté ». Elle relie cette « victoire » à sa « force de caractère », et explique en ligne à une autre femme à qui l'intervention a été refusée que « le médecin a peut-être senti qu'il y avait quelque chose de pas décidé chez toi $\gg{ }^{17}$.

Les femmes interrogées se livrent peu à la psychologisation de leur choix contraceptif ${ }^{18}$. Cela a de quoi étonner dans une société où le regard psychologique sur soi est assez répandu et où les femmes interrogées par d'autres sociologues sur le choix de non-maternité ont opéré cette introspection ${ }^{19}$. Face à un gouvernement des corps qui use de leur psyché contre elles, elles se posent comme des individus sans profondeur psychologique, purement volontaires et rationnels : comme des « moi » sans zone d'ombre.

\section{La stérilisation : un double régime de justification}

Déterminées à convaincre leur interlocuteur-le médecin-, les femmes interrogées ont produit des justifications à leur choix. Celles-ci contiennent deux demandes associées : une demande de naturel en tension avec une demande de suppression radicale de la fertilité.

\section{Une demande de naturel}

La volonté de ne plus vouloir supporter la contraception est constamment évoquée. La conservation de la fertilité a en effet un coût, présenté comme injustifié chez une femme ne souhaitant pas d'enfant : les inconvénients de la contraception. Les enquêtées relatent souvent un parcours contraceptif long, présenté négativement. Ce parcours comprend des essais de plusieurs types de pilule, du stérilet, voire de l'implant. Au contraire de la stérilisation qui est présentée comme libératrice, source d'indépendance, la contraception non définitive est associée à un lexique dépréciatif

${ }^{16}$ L. GuYARD, « Chez la gynécologue. Apprentissage des normes corporelles et sexuelles féminines », Ethnologie française, vol. 40, 2010/1, p. 67-74.

17 On peut lire dans cette formulation une certaine psychologisation de la décision de stérilisation, en contradiction avec ce qui suit. Cependant, la phrase ne dénote pas d'une volonté de cerner les motifs inconscients d'un choix de se faire stériliser : elle pointe du doigt l'indécision d'une autre femme.

${ }^{18}$ Certaines femmes sur le groupe childfree parlent néanmoins de «phobie de la grossesse», référant au caractère psychiquement insupportable de leur fertilité. La stérilisation est présentée comme une solution, précisément pour conserver leur intégrité psychique - elles font ici écho aux discours médicaux mentionnant la fertilité comme essentielle à la santé psychologique, mais retournent l'argumentation.

19 Ch. Debest, Le choix d'une vie sans enfant, op. cit.; A. Gotman, op. cit. 
référant à la contrainte, critiquée comme « non naturelle » (la pilule), ou intrusive (le stérilet) :

F4 : Moi c'est sûr que ça me faisait chier, hein, les hormones. Enfin je veux dire c'est hyper mauvais, moi j'avais peur que ça me provoque... Je sais pas, enfin, sachant que je veux pas d'enfant, 20 ans d'hormones sur mon corps... Ils disent que ça fait rien, que ça diminue le risque de cancer de je sais pas quoi... Moi je sais juste que je veux pas prendre ça. Ça m'angoisse.

F6 : Après moi les hormones ça m'a fait gonfler, déjà que j'étais pas... J'ai pris dix kilos en plus. Et j'avais des migraines.

F 9 : Pourquoi je voulais pas de stérilet, euhh, bah parce que ça me dérangeait d'avoir un corps étranger dans mon corps, voilà, enfin vraiment l'idée me dérangeait ${ }^{20}$.

On peut inscrire cette volonté de «naturel » dans un contexte plus général de « crise de la pilule ${ }^{21}$, remise en cause depuis 2012-2013 avec les affaires des pilules de $3^{\mathrm{e}}$ et $4^{\mathrm{e}}$ générations. Les opinions négatives à propos de la pilule sont de plus en plus répandues, surtout chez les jeunes générations (44\% d'opinions favorables à la pilule en 2010 contre $31 \%$ en $2013^{22}$ ).

\section{En finir avec la fertilité : une demande radicale}

Une demande très spécifique aux childfree est la volonté d'en finir avec la fertilité, à propos de laquelle les enquêtées expriment lassitude, voire angoisse. Le thème du corps qui trahit est fréquemment évoqué. La possibilité du déni de grossesse comme facteur d'angoisse a été évoquée deux fois dans nos entretiens, et est revenue quelques fois dans les postes du groupe childfree francophone que nous suivions. Comme celui-ci : «Que feriez-vous si vous faisiez un déni de grossesse ? », poste suscitant réponses effrayées et solutions radicales : « je le mettrais au congélo » commente une femme sur le ton de l'humour. F2 parle en entretien du déni de grossesse de sa sœur qui l'a marquée et l'a poussée plus encore à envisager une stérilisation :

F2 : Ma sœur a fait un déni de grossesse. Elle a dit que c'était un déni de grossesse et la gamine est arrivée le 25 décembre... Donc euh voilà, presque sans prévenir. Donc du coup, euh oui ça fait un petit peu peur. On voit plein d'émissions de chais pas quoi à la télé, comment un jour un bébé qui devait pas être là et qui est là, comment le corps humain est tellement, fait tellement peur... Et est tellement fascinant parce que qu'il se passe plein de choses dedans. J'avais vraiment peur de ça... un jour de me retrouver maman malgré moi, dans ces cas-là tu te dis qu'est-ce que je fais, je tue l'enfant, je me tue moi ?

Cette urgence à se séparer de sa fertilité est d'autant plus présente que celle-ci est contraignante : c'est ainsi particulièrement le cas chez F11, " hyperfertile ». Ce corps qui ne répond pas à la volonté de la femme est parfois source de griefs :

${ }^{20}$ Ce refus des « corps étrangers » est fréquent chez les femmes interrogées, et fréquent chez les femmes en général selon le Dr 5 .

${ }^{21}$ N. Bajos, M. Rouzeaud-Cornabas, H. Panjo et alii, « La crise de la pilule. Vers un nouveau modèle contraceptif? », Population et Sociétés, $n^{\circ}$ 511, 2014.

${ }^{22}$ La question était «Pensez-vous que la pilule permet aux femmes d'avoir une sexualité plus épanouie? ». 
une internaute déclare ainsi ressentir comme une " insulte » (poste sur un groupe childfree) qu'il faille « tromper (leur) corps pour l'empêcher de faire des enfants ". Le corps fertile, procréateur, est parfois une source de dégoût. Faisant référence à la grossesse, une internaute affirme : « ça me dégoûte, je trouve ça laid, ça m'a jamais attirée ». D'autres partagent des photos d'accouchement apocalyptiques. À une pression sociale à utiliser leur fertilité, les femmes childfree répondent par la volonté de la supprimer définitivement, par un refus par corps de la maternité ${ }^{23}$. En creux, on lit une revendication de la liberté du choix child-free ${ }^{24}$ : libérées des exigences de la maternité, ces femmes le sont également de tout ce qui les y rattache corporellement.

Cette dernière demande les place dans la droite lignée du mouvement de « refus du corps comme destin » ${ }^{25}$ sensible à partir des années 1960 et surtout 1970 et caractérisé par la mise à disposition d'un nombre croissant de moyens de contrôle sur son corps : contraception, avortement, euthanasie dans certains pays.

Elle les place aussi dans une perspective pleinement déviante rapportée aux normes de genre qui font de la maternité un élément constitutif de la féminité. De la même manière que la lesbienne ${ }^{26}$, la femme sans enfant stérilisée «n'est pas une femme ${ }^{27}$. Elle fait défi de la norme qui enjoint de procréer mais aussi d'être fertile, pour être femme. Témoin de cette norme en médecine en médecine, la vérification annuelle du bon état de la machinerie féminine dès la puberté, qui place le gynécologue au rang du spécialiste le plus consulté par les femmes ${ }^{28}$. De nombreuses femmes rapportent aussi des injonctions à se conformer à la norme de procréation, qui sont aussi des injonctions à se conformer aux normes de genre : « l'épanouissement d'une femme, ce sont ses enfants », " elles disent souvent ça, mais elles changent d'avis ».

Il ne s'agit donc pas seulement de refuser son destin corporel, mais aussi de refuser d'être «plus corps que l'homme ${ }^{29}$. La liberté offerte par la stérilisation est donc une liberté qui rejoint celle des hommes, non chargés par la contraception. Cette demande radicale est aussi un refus de voir son individualité résumée à la fertilité.

${ }^{23}$ Si la lassitude de la fertilité est bien présente, elle ne remonte souvent qu'à une dizaine d'années, comparée au choix de ne pas vouloir d'enfants, daté bien souvent à l'enfance de la femme («Déjà moi quand j'étais petite ben ça m'a jamais attirée du tout ! J'ai jamais eu l'idée de me dire « bah tiens je vais faire un gosse », jamais. » affirme F2). Les femmes childfree semblent se conformer dans un premier temps à l'impératif de la contraception réversible, se lançant dans un parcours contraceptif chaotique. Une fois les contraintes de la contraception explorées, elles formulent ainsi cette lassitude, qui vient redoubler celle d'avoir à " prendre » une contraception inutile.

${ }^{24}$ A. Gotman, op. cit.

25 D. Memmi, La Revanche de la chair, Paris, Seuil, 2014, p. 251.

${ }^{26}$ Toute proportion gardée dans la comparaison.

27 M. Wittig, La Pensée straight, Paris, Éditions Amsterdam, 2007, p. 61.

${ }^{28}$ L. Ruault, « La force de l'âge du sexe faible. Gynécologie et construction sociale d'une vie féminine », Nouvelles Questions Féministes, vol. 34, 2015/1, p. 35-50.

${ }^{29}$ D. Le Breton, La sociologie du corps, Paris, Presses universitaires de France, 2008, p. 108. 


\section{La stérilisation, un acte chargé symboliquement}

La possession de moyens permettant de remettre en cause les normes contraceptives, l'adoption de stratégies de contournement, le regroupement en communauté partageant les mêmes valeurs permettent finalement aux childfree d'investir un acte pourtant largement impensé par la profession médicale. L'enjeu est donc ici de rendre pensable la stérilisation.

La stérilisation tient une place toute particulière dans la «culture chidfree ». Les membres du groupe ont même développé une lexicologie particulière vis-à-vis de celle-ci : la boutade « je suis libérée, délivrée » signifie ainsi « je me suis fait stériliser ». Une femme commente, sous une publication sur la stérilisation : « Moi aussi bientôt je serai libérée, délivrée ! ».

\section{Un marqueur corporel de l'intensité du choix}

Choix radical, la stérilisation est une marque laissée sur son corps. Marque qui vaut au moins à trois niveaux : vis-à-vis des autres childfree, vis-à-vis de l'entourage, et vis-à-vis de soi.

Vis-à-vis des autres childfree, ce geste est souvent revendiqué avec fierté, en ligne. Il a valeur d'exemple pour encourager les autres qui souhaiteraient effectuer cette démarche, mais sert aussi la valorisation de soi et de sa détermination. Il signifie l'impossible retour en arrière et s'oppose au thème récurrent du changement d'avis : les publications présentent de nombreux récits de childfree faisant finalement des enfants.

Vis-à-vis de l'entourage, il constitue surtout une protection contre la remise en cause du choix. La stérilisation fait progresser la femme dans sa « carrière déviante ${ }^{30}$, mais elle lui donne aussi les moyens de faire reconnaître son choix. Par son caractère définitif, elle donne une gravité au choix de ne pas avoir d'enfant, comme le montre le discours de cette enquêtée :

F12 : On me gonfle ouais, je vais en parler (du fait qu'elle est stérilisée autour d'elle). Si on me met trop la pression là-dessus là j'en parlerai [...] Ce serait mon plaisir quoi, de voir leur tête. Parce que moi j'adore provoquer aussi donc...

Cette gravité vaut également dans le cadre de la rencontre amoureuse, au moins hétérosexuelle. De nombreuses childfree évoquent sur le groupe, ou en entretien, les rencontres avec des hommes qui croient leur non-désir d'enfant transitoire. Or une stérilisation « annonce tout de suite la couleur » (F8). Il semblerait donc qu'avec la stérilisation on passe d'un âge social à un autre : de l'irresponsabilité et de l'indécision propre à une femme n'ayant pas eu d'enfant ${ }^{31}$ elle fait passer ${ }^{32}$ à la maturité symbolique de celle dont les capacités reproductives sont épuisées.

C'est aussi et surtout dans le récit de soi (pour soi) que la stérilisation prend une place importante. Les femmes l'évoquent d'elles-mêmes comme un événement

${ }^{30}$ H. BeCKer, Outsiders, Paris, Metaillé, 1985 [1963].

${ }^{31}$ L. Ruault, « La force de l'âge du sexe faible. Gynécologie et construction sociale d'une vie féminine $», l o c$. cit.

${ }^{32}$ La ménopause est cependant souvent présentée comme un déclassement accompagné de souffrances psychiques pour une femme, ibid. 
important, « libérateur » (F7) et nous parlent spontanément du moment où elles ont commencé à y penser. Il s'agit là de transformer un statut passif (ne pas encore avoir fait d'enfant) en un choix actif (ne jamais faire d'enfant). En d'autres termes, il s'agit de passer de childless à childfree ${ }^{33}$, c'est-à-dire du « lexique de la privation » à celui de la liberté ${ }^{34}$. On parle bien ici d'acquérir un «titre » ${ }^{35}$ et ainsi de s'arroger une part de la victoire symbolique d'avoir réussi à se nommer. Devenir childfree amène la relecture de sa biographie individuelle à partir de cette catégorie collective, aussi la stérilisation tient-elle une place plus qu'importante dans le récit de vie, comme pour cette enquêtée citée en introduction, qui affirme se faire stériliser « pour ses trente ans ». Il s'agit là d'une mise en scène de l'acte : à cet âge où les femmes font usuellement des enfants, l'idée est d'aller à contre-courant.

\section{Une forme d'autocontrôle radical}

«Libération » pour ces femmes, la stérilisation peut cependant être vue comme une forme d'autocontrôle radical de la fertilité. Contrôler sa fertilité n'est pas en soi exceptionnel, le recours aux méthodes anticonceptionnelles est aujourd'hui la norme : $90,2 \%$ des femmes non stériles, non enceintes, ayant un partenaire homme et ne cherchant pas à avoir un enfant utilisent une contraception (Baromètre Santé, 2010). De la première prescription de pilule à la ménopause, la fertilité est scrutée, évaluée, bref contrôlée par ces experts du corps féminin que sont les gynécologues. La profession valorise la fertilité auto-contrôlée par une bonne gestion contraceptive, sous sa supervision attentive ${ }^{36}$.

S'ils parlent de libération du corps, les childfree ont pourtant intégré et reconnaissent la norme de contrôle de la fertilité ${ }^{37}$. Ils dénoncent ainsi largement le manque d'auto-contrôle reproductif, et font la critique de certaines figures repoussoirs. Parmi lesquelles : les « Papounes » et de « Mamounes », parents « un peu bêtes, un peu gagas » (F9) dévolus à leur « enfant-roi ». La figure de la mauvaise famille est souvent évoquée : non seulement parce qu'ils procréent trop, mais aussi parce qu'ils procréent « mal » : nombre de publications dénoncent l' « intrusion » d'enfants « mal élevés », « bruyants », " débiles » dans le quotidien. La mauvaise tenue est aussi parfois une mauvaise tenue sexuelle : la «Mamoune » est parfois assimilée à la putain. « Il a bien fallu qu'elle l'écarte ses cuisses (sic) », « Les mamans sont les plus grosses salopes, je vois pas pourquoi dès qu'on fait un gosse on oublie le processus de fabrication » commente une childfree. Vraie stigmatisation des conduites sexuelles ou retournement ironique d'un stigmate - les childfree se faisant souvent les défenseurs de la liberté sexuelle ? Cette mauvaise tenue de soi et cette mauvaise éducation sont

${ }^{33}$ On peut expliquer de cette manière la place que tient la stérilisation chez les childfree : se faire stériliser c'est véritablement devenir childfree.

${ }^{34}$ A. Gotman, op. cit.

35 Ibid.

${ }^{36}$ L. Ruault, «La force de l'âge du sexe faible. Gynécologie et construction sociale d'une vie féminine », loc. cit.

${ }^{37}$ Celle qui consiste en tout cas aujourd'hui à maîtriser sa fécondité par le biais de la contraception, ainsi qu'à choisir le « bon » moment pour procréer (avoir un conjoint, un emploi stable...). 
en tous les cas autant de conduites qui s'opposent à l'idéal de « civilisation » dont parle N. Elias ${ }^{38}$.

Si les childfree souhaitent contrôler leur fertilité, la vraie différence est qu'elle cherchent à le faire de manière plus efficace, radicale. Est requise l'assimilation complète de cette norme d'autocontrôle reproductif par leur corps, gage pour elles de l'indépendance vis-à-vis de la contraception. L'effet recherché est donc que la norme devienne naturelle au corps : privé de ses capacités reproductives, il est totalement contrôlé, docile. Puisqu'il n'est plus besoin de passer par des contraintes extérieures pour contrôler la fertilité, la stérilisation constitue une forme d'autocontrôle. Se faire stériliser, c'est donc sortir de la soumission à l'arbitraire du corps : c'est être plus civilisé que son voisin, « lapin », « mal éduqué » à la fertilité envahissante.

\section{Civilisation des moeurs reproductives, ou mépris de classe?}

Il apparaît pertinent de parler de mépris de classe de certains childfree quand la fertilité non contrôlée est présentée de manière extrêmement négative, et est associée à des caractéristiques sociales stigmatisantes. Les familles trop nombreuses se voient ainsi ironiquement taxer de « lapins », ou « cas sociaux ». Les prénoms sont aussi régulièrement moqués. Les moqueries les plus fréquentes concernent les prénoms anglo-saxons ou des prénoms à caractère fortement « innovant »: « Mamoune se promène avec Djaÿson » peut-on par exemple lire sous un post childfree. Ces prénoms sont dans leur grande majorité des prénoms de classe populaire ${ }^{39}$. Sous la dénonciation du caractère « ridicule » des prénoms se cache donc également une dénonciation des caractéristiques sociales de leurs porteurs. À ces " mauvais goûts » culturels des « Parounes $»^{40}$ s'ajoute une « mauvaise tenue de soi » : les postes childfree dénoncent de manière récurrente la mauvaise éducation des enfants, envahissants et bruyants. Parfois est évoquée l'odeur gênante de l'enfant : « Je ne veux pas qu'elle le change chez moi ça me dégoûte ", "Je comprends pas comment on peut essuyer le vomi de son chiard». Être confronté aux sécrétions corporelles de l'enfant (vomi, urine, selles) est ici présenté comme un avilissement. On pourrait, dans une certaine mesure, faire le rapprochement avec le rôle social de l'odorat dont parle Alain Corbin ${ }^{41}$ : les familles sont présentées comme reléguées dans le domaine du sale, du putride, du malsain.

La procréation est également la norme dans les classes supérieures, et il serait bien sûr réducteur d'assimiler totalement les valeurs du groupe childfree à des valeurs de classe. Cependant, ce contrôle de la fertilité apporté par la stérilisation pourrait bien être un marqueur social. C'est un attribut de classe supérieure qui sait mettre à distance le bas corporel (vomi, urines, selles), mais aussi l'instinct maternel (celui des « Parounes », tant moqués), et enfin le corps procréateur et ce qu'il engendre. Les childfree enseignent en définitive quoi faire avec son corps : savoir l'utiliser

${ }^{38}$ N. Elias, La Civilisation des Mours, Paris, Calmann-Lévy (coll. « Liberté de l'esprit »), 1991 [1939].

39 B. Coulmont, Sociologie des prénoms, Paris, La Découverte (coll. « Que sais-je ?»), 2011.

40 « Parounes » désigne le couple parental « Papoune » et « Mamoune ».

${ }^{41}$ A. Corbin, Le miasme et la jonquille. L'odorat et l'imaginaire social, XVIII ${ }^{e}$-XIX $X^{e}$ siècle, Paris, Flammarion (coll. « Champs Histoire »), 1982. 
à des usages présentés comme plus épanouissants que la procréation, et dans une certaine mesure comme plus légitimes ${ }^{42}$. La volonté de distinction, qui consiste à « aimer pareillement d'autres choses » mais aussi à " aimer autrement des mêmes choses ${ }^{43}$ est bien sensible dans la demande de stérilisation : se faire stériliser, c'est aimer différemment le même corps, et trouver le même plaisir à faire d'autres choses avec (« se réaliser » plutôt que faire des enfants).

\section{Conclusion}

La stérilisation, bien que "déviante », est de moins en moins secrète. Elle fait l'objet d'un traitement médiatique où certaines femmes témoignent à visage découvert ${ }^{44}$. En entretien les femmes interrogées parlent ouvertement de leur stérilisation, sans s'excuser, contrairement par exemple à l'avortement ${ }^{45}$. Ce sont leurs « armes sociales » ${ }^{46}$, mais aussi leur regroupement, qui permettent à ces femmes sans enfant de revendiquer l'accès à la stérilisation volontaire, ainsi que de l'investir symboliquement. Nous choisissons d'insérer ce phénomène dans une dynamique plus générale : celle de l'auto-contrôle croissant des pulsions corporelles ${ }^{47}$, ici reproductives.

La stérilisation au cœur d'un réseau de contre-normes. Il ne s'agit finalement pas que de donner un sens à la stérilisation : nous pouvons replacer la stérilisation au cœur d'un réseau de contre-normes portées par le mouvement childfree. Celles-ci reposent sur un renversement des valeurs associées à la procréation. Cela constitue un bouleversement total dans une société accordant encore une grande valeur à la procréation, et assimilant procréation et filiation ${ }^{48}$. Les childfree refusent ce modèle de société : ils refusent la procréation, ainsi que son couplage avec la filiation. Dans le cas où un individu voudrait absolument des enfants, l'adoption apparaît comme un choix «plus moral » ${ }^{49}$, pour raisons économiques, ou écologiques : « De toutes les manières si je voulais absolument un enfant plus tard, j'adopterais, je trouve ça mieux » (F11). Dans une société où l'adoption et les modes de procréation alternatifs (PMA) sont encore largement tenus de respecter l'apparence de la procréation conjugale, les childfree vont totalement à contre-courant.

${ }^{42}$ On peut lire ainsi la critique dirigée vers les femmes au foyer, qui « ne savent pas se réaliser autrement que par un enfant ».

${ }^{43}$ P. Bourdieu, La Distinction. Critique sociale du jugement, Paris, Éditions de Minuit (coll. « Le Sens Commun »), 1979.

${ }^{44}$ Voir le webdocumentaire « J'ai décidé d'être stérile », H. Rocco et alii.

${ }^{45}$ A.-S. DivaY, «L'avortement : une déviance légale », Déviance et Société, vol. 28, 2004, p. 195-209.

${ }^{46}$ Ch. Debest, Le choix d'une vie sans enfant, op. cit.

${ }^{47}$ N. Elias, La Civilisation des mours, op. cit.

48 I. THÉRY, Des humains comme les autres. Anonymat et genre du don, Paris, EHESS, 2010, p. 180.

${ }^{49}$ Notamment pour des raisons écologiques et de surpopulation. 
On assiste donc à un « retournement du stigmate ${ }^{50}$, qui ne conduit pas seulement à valoriser ses propres choix, mais à stigmatiser les stigmatiseurs ${ }^{51}$. Ainsi, à l'accusation d'égoïsme faite aux childfree répond l'accusation d'égoïsme faites aux parents : faire en enfant pour « toucher des allocations », pour ne pas être seul dans la vieillesse ou pour avoir un « mini-moi » sont autant de preuves d'un égoïsme qui ne dit pas son nom. Au devoir social de faire des enfants répond un devoir social de ne pas en faire. Le travail est par exemple particulièrement valorisé au sein de la communauté childfree. Reprenant l'accusation éculée de l'assistanat social ${ }^{52}$, ils la dirigent vers les familles : les «Papounes » et surtout les « Mamounes » sont ceux qui profitent des aides sociales sans travailler. Les publications sur le groupe childfree consistent souvent en des partages d'articles sur des faits divers familiaux, affaires d'inceste, fils ayant violé sa mère, mère profitant des aides sociales indûment... Ce grand récit collectif alimente la dénonciation de l'« hypocrisie » des valeurs morales accordées à la famille.

Dans ce qu'ils considèrent comme leur défouloir, nombre de childfree adoptent une rhétorique de l'excès. Leurs revendications s'accompagnent d'une rhétorique provocante qui appelle par dérision au défi des normes morales : une plaisanterie récurrente suggère ainsi de «mettre des bébés dans le congélo " $^{53}-$ ou de manière plus générale à se débarrasser violemment des " gosses » des autres. Les childfree tutoient parfois l'eugénisme, sur un mode ironique (« on devrait les stériliser, ces cassos ! »), ou sur un mode plus sérieux : ils condamnent parfois moralement le fait de faire un enfant alors que l'on est atteint d'une maladie génétique, ou réfléchissent sur l'instauration d'un « permis d'enfanter $»^{54}$.

${ }^{50}$ Ch. Debest, Le choix d'une vie sans enfant, op. cit.

${ }^{51} \mathrm{Ou}$ tout du moins ce qui est perçu comme les stigmatiseurs c'est-à-dire essentiellement ces figures de la « norme procréative » que ce sont les « Parounes».

52 P. Rosanvallon, La crise de l'Etat providence, Paris, Seuil, 1981.

${ }^{53}$ Plaisanterie faisant référence à l'affaire des bébés congelés (affaire Véronique Courjault).

${ }^{54}$ La qualification d' « eugénisme » est bien sûr compliquée à appliquer ici - raison pour laquelle nous préférons affirmer que certains propos tutoient l'eugénisme, manière d'indiquer que nous ne savons pas s'ils s'y rapportent tout à fait, mais qu'ils nous y font un peu penser. Ici, en effet, il n'y a pas là un projet cohérent et organisé de mise en ordre de la transmission génétique. Il y a en revanche l'idée que certains auraient plus de qualités les autorisant à se reproduire que d'autres. Par ailleurs, comme le souligne Paul-André Rosental, dans sa réflexion sur le legs contemporain de l'eugénisme (P.-A. Rosental, Destins de l'eugénisme, Paris, Seuil, 2016), l'eugénisme ne se résume pas seulement à une dimension coercitive. Selon lui, certains et notamment les médecins défendant la pratique diagnostic pré-natal - opposent un eugénisme $\mathrm{du}$ « libre-choix », à un eugénisme « négatif », qui serait quant à lui autoritaire, marqué par la volonté d'empêcher les « nuisibles» de se reproduire. L'idée est que si les individus dont la reproduction n'est pas jugée « souhaitable » choisissent eux-même de ne pas se reproduire, il n'y a pas de problème, et aucune commune mesure avec ce «mauvais eugénisme » autoritaire dont on a fait par exemple l'expérience dans les régimes totalitaires du premier $\mathrm{XX}^{\mathrm{e}}$ siècle. Ce point de vue peut se défendre, mais selon P.-A. Rosental, c'est oublier un peu vite le poids des normes sur les individus : formuler des règles en matière de qui a ou non le droit, moralement, de se reproduire, est déjà tenter un peu de contraindre certains. La sanction pour celui qui se 
Le projet childfree est marqué par la volonté de contrôler radicalement la nature en figeant son développement propre. L'appel à se faire stériliser, à « instaurer un permis d'enfanter » en sont symptomatiques. On retrouve ici d'autres indices de ce procès de civilisation que Dominique Memmi a constaté non seulement autour de la naissance mais aussi de la mort, qui en seraient aujourd'hui selon elle des domaines privilégiés ${ }^{55}$. Ces groupes sociaux seraient, de ce point de vue, bien à la pointe du procès de civilisation portant sur la prolifération organique, sur le développement normal, « naturel », de la corporéité ${ }^{56}$.

Autocontrôle, ou normalisation du corps féminin ? S'il s'agit bien d'un autocontrôle, la stérilisation constitue cependant la forme d'un autocontrôle spécifiquement féminin. Sur les groupes childfree, la charge de la critique de la parentalité est majoritairement laissée aux femmes et dirigée contre les femmes. Ces mères sont qualifiées de « pondeuses » ou de "lapines » autant de caractéristiques genrées qui animalisent le corps féminin (sur)procréateur. Certaines childfree se revendiquent d'un féminisme " antimaternité », valorisant particulièrement le travail féminin et stigmatisant inversement la « dépendance » des mères au foyer. Le thème de l'absence d'autonomie se décline essentiellement contre ces femmes qui ne savent pas gérer leur fertilité, procréent trop ou procréent mal. Sous la vidéo d'une femme enceinte se déhanchant sur de la musique latino, postée sur le groupe par une internaute « dégoûtée : elles ont vraiment besoin de nous montrer leur gros ventre ? », une autre répond : « au moins elle reste active pendant sa grossesse, ça change ! »

La stérilisation est rarement envisagée comme solution masculine : sur le groupe, les hommes peu nombreux parlent peu de vasectomie. Si la stérilisation est libératrice, c'est avant tout pour les femmes. Les childfree, inspirées par les récents débats autour de la « charge mentale », parlent de « charge contraceptive » incombant aux femmes, et que la stérilisation permettrait de soulager. Il n'en reste pas moins que les femmes supportent seules cette intervention, rarement accompagnées de leurs éventuels conjoints. Les tentatives de certains médecins pour défendre la stérilisation masculine, présentée comme moins invasive, tombent souvent court : soit parce que l'homme n'envisage pas de se faire stériliser, mais aussi et surtout parce que les childfree conçoivent la stérilisation comme une décision sans rapport avec le conjoint, toute personnelle.

Cet acte s'inscrit donc une nouvelle fois, mais sous une forme radicale, dans la logique de normalisation du corps féminin et de domestication de la fertilité féminine qui laisse majoritairement aux femmes la charge de la contraception. Si les childfree subvertissent bien certaines normes, ce ne sont donc pas totalement celles, multiples et complexes, du genre.

reproduit sans y avoir « le droit » n'est alors plus légale, mais sociale - constitue-t-elle parfois une contrainte presque comparable?

55 D. Meмmi, Faire vivre et laisser mourir. Le gouvernement contemporain de la naissance et de la mort, op. cit.

${ }^{56}$ D. Memmi, La Revanche de la chair, op. cit. 


\section{Bibliographie}

Bajos N., Rouzeaux-Cornabas M., Panjo H. et alii, " La crise de la pilule en France : vers un nouveau modèle contraceptif? », Populations et Sociétés, ${ }^{\circ} 511$, mai 2014.

Becker H., Outsiders, Paris, Metaillé, 1985 [1963].

Blanc B. et Madelenat P., La stérilisation à visée contraceptive, Paris, Elsevier Masson, 2004.

Boltanski L., Chiapello E., Le nouvel esprit du capitalisme, Paris, Gallimard, 1999.

Bourdieu P., La Distinction. Critique sociale du jugement, Paris, Éditions de Minuit, 1979.

Bretin H., Contraception : quels choix pour quelle vie ? Récits de femme, paroles de médecins, Paris, INSERM, 1992.

Corbin A., Le miasme et la jonquille. L'odorat et l'imaginaire social : XVIII ${ }^{e}$ XIX siècle, Paris, Flammarion, 1982.

Coulmont B., Sociologie des prénoms, Paris, La Découverte, 2011.

DeBest Ch., Le choix d'une vie sans enfant, Rennes, Presses universitaires de Rennes, 2014.

Dingwall R. et Murray T., "Categorization in accident department: "Good » patients, " $\mathrm{Bad}$ » patients and « Children » », Sociology of Health and Illness, 5 (2), 1983, p. 127-148.

Divay A.-S., « L'avortement : une déviance légale », Déviance et Société, vol. 28, 2004, p. 195-209.

Donati P., « L'absence d'enfant. Un choix plus ou moins délibéré dans le parcours d'hommes et de femmes ", Revue des politiques sociales et familiales, $\mathrm{n}^{\circ} 62$, 2000, p. 43-56.

Elias N., La Civilisation des mours, Paris, Calmann-Lévy (coll. " Liberté de l'esprit »), 1991 [1939].

Giami A. et Leridon H., Les enjeux de la stérilisation, Paris, INED Éditions, 2000.

Giddens A., La transformation de l'intimité. Sexualité, amour et érotisme dans les sociétés modernes, trad. Jean Mouchard, Paris, La Rouergue/Chambon, 2004.

Goffman E., Stigmates. Les usages sociaux des handicaps, Paris, Éditions de Minuit, 1975.

Gotman A., Pas d'enfant. La volonté de ne pas engendrer, Paris, Éditions de la Maison des Sciences de l'Homme, 2017.

GuYARD L., « Chez la gynécologue. Apprentissage des normes corporelles et sexuelles féminines », Ethnologie française, vol. 40, 2010/1, p. 67-74.

Honneth A., La lutte pour la reconnaissance, Paris, Folio, 2013.

Le Breton D., La sociologie du corps, Paris, Presses universitaires de France, 2008.

Madelenat P. et Blanc B., La stérilisation à visée contraceptive, Paris, Elsevier Masson, 2004.

Mazuy M., Debest Ch. et alii, « Rester sans enfant. Un choix de vie à contrecourant », Population et Sociétés, ${ }^{\circ}$ 508, 2014.

Memmi D., La Revanche de la chair, Paris, Seuil, 2014.

Meмmi D., Faire vivre et laisser mourir, Le gouvernement contemporain de la naissance et de la mort, Paris, La Découverte, 2003. 
Memmi D. et Fassin D. (dir.), Le gouvernement des corps, Paris, Éditions de l'EHESS, 2004.

Rosanvallon P., La crise de l'Etat providence, Paris, Seuil, 1981.

Rosental P.-A., Destins de l'eugénisme, Paris, Seuil, 2016.

RuAult L., « La force de l'âge du sexe faible. Gynécologie et construction sociale d'une vie féminine », Nouvelles Questions Féministes, vol. 34, 2015/1, p. 35-50.

TAвет P., Fécondité naturelle, fertilité forcée, in La construction sociale des inégalités de sexe, Des outils et des corps, Paris, L'Harmattan, 1998.

Thery I., Des humains comme les autres. Anonymat et genre du don, Paris, EHESS, 2010.

WitTig M., La Pensée straight, Paris, Éditions Amsterdam, 2007. 